\title{
Ghost cell odontogenic cyst: A rare case with literature review
}

\author{
B.N. Shivakumar ${ }^{1}$, Santhosh Reddy ${ }^{2}$, Barakha Nayak ${ }^{3}$, Nehal Rathore ${ }^{4}$ \\ ${ }^{1}$ Professor \& HOD, ${ }^{3}$ Reader, ${ }^{4}$ Post Graduate Student, Department of Oral \& Maxillofacial Pathology, Maitri College of Dentistry \& Research \\ Centre, Anjora, Durg - 491001, Chhattisgarh, India, ${ }^{2}$ Reader, Department of Oral \& Maxillofacial Pathology, Rungta College of Dentistry, \\ Bhilai, Chhattisgarh, India
}

\section{A B S TR A C T}

Gorlin in 1962 described calcifying odontogenic cyst (COC) for the first time as a separate entity. It is an odontogenic cyst with notable presence of histopathological features which contains a cystic lining demonstrating "Ghost" cells with a propensity to calcify. COC shows diversity of variants according to its clinical, histopathological \& radiological characteristics. A case of calcifying odontogenic cyst is reported with its review of literature.

Key words: Calcifying odontogenic cyst, Odontogenic cyst, Ghost cells
Access this article online

Website:

http://nepjol.info/index.php/AJMS

DOI: $10.3126 / a j m s . v 6 i 4.11586$

\section{INTRODUCTION}

In clinical situations, unusual presentation of uncommon lesion or early presentation of rare lesions can mimic routine pathological entities. ${ }^{1}$ The signs and symptoms of such lesions are often misleading and pose a diagnostic and therapeutic challenge, uncommonly; rare pathologies mimic the commonly encountered typical periapical lesions. ${ }^{1}$

The calcifying odontogenic cyst (COC) was first characterized by Gorlin et al. ${ }^{2}$ COC is now recognized as a distinct pathologic entity by the World Health Organization. ${ }^{3}$ The COC is generally regarded as a benign lesion of odontogenic origin, which can present as a cyst or as a neoplasm. ${ }^{3} \mathrm{COC}$ is usually asymptomatic and may be an incidental radiographic finding. ${ }^{3}$

In coincidence a case of calcifying odontogenic cyst is reported with brief review of literature, in 26 years old female, which was an incidental radiographic finding, without any obvious swelling and associated symptoms.

\section{CASE REPORT}

A 26 year old female reported with chief complaint of spacing and proclination of her teeth in maxillary front teeth region (Figure 1). Patient is undergoing orthodontic treatment for the same. Intraoral examination revealed a retained deciduous canine 53 on right maxilla, which was showing grade I mobility (Figure 2). Oral hygiene status of patient is satisfactory and no abnormalities were noted. Extraction 53 was planned.

On radiographical examination, Intra-Oral Periapical Radiograph (IOPA), Occlusal \& OPG shows a well circumscribed radiolucency over the retained deciduous canine and impacted permanent canine (Figures 3-5).

The extraction of 53 and curettage of extraction socket was done. The soft tissue pieces obtained, were sent for histopathological examination, with a provisional diagnosis of radicular cyst.

On histopathological examination, it was found that the lesional tissue is composed of fibrous capsule with a lining of odontogenic epithelium. The basal cells of the epithelial lining are columnar in shape similar to ameloblasts. The overlying layer of loosely arranged epithelium shows stellate reticulum like cells. Variable number of "ghost cells" were present within the epithelium. The fibrous capsule is composed of densely packed collagen fibres \& numerous chronic inflammatory cells seen dispersed between blood vessels (Figures 6 and 7). Based on the histopathological findings, the final diagnosis of COC 


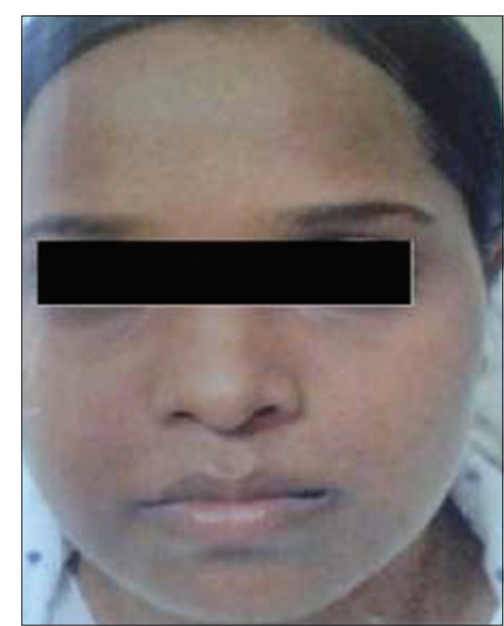

Figure 1: Facial view of the patient

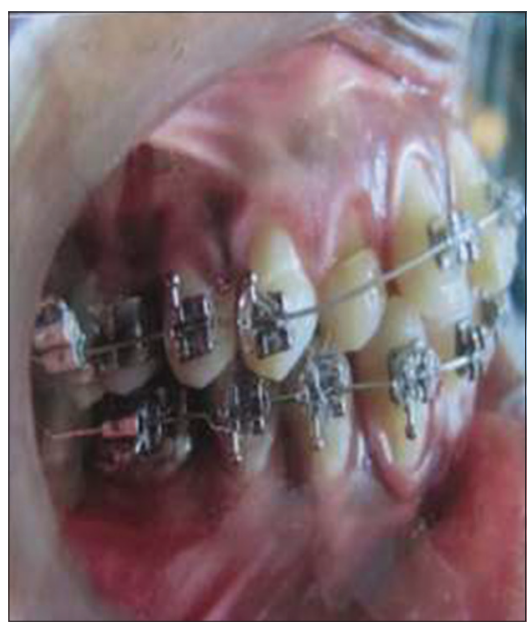

Figure 2: Intra-oral view showing retained deciduous teeth without any associated swelling

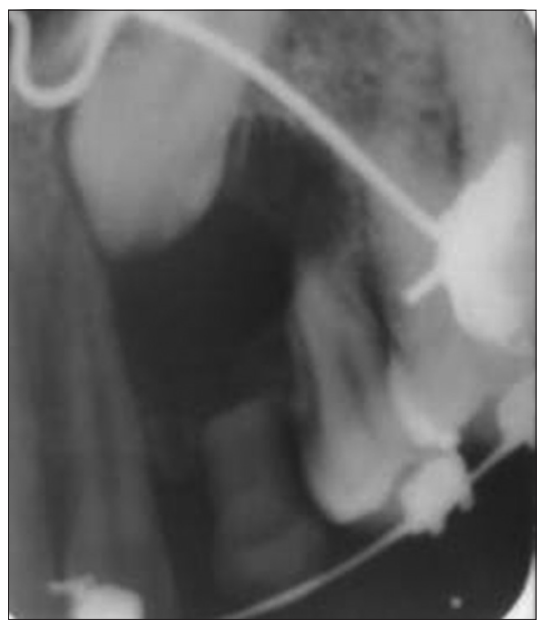

Figure 3: Introral periapical radiograph showing well defined periapical radiolucency associated with retained deciduous canine retained deciduous canine, impacted canine on right side

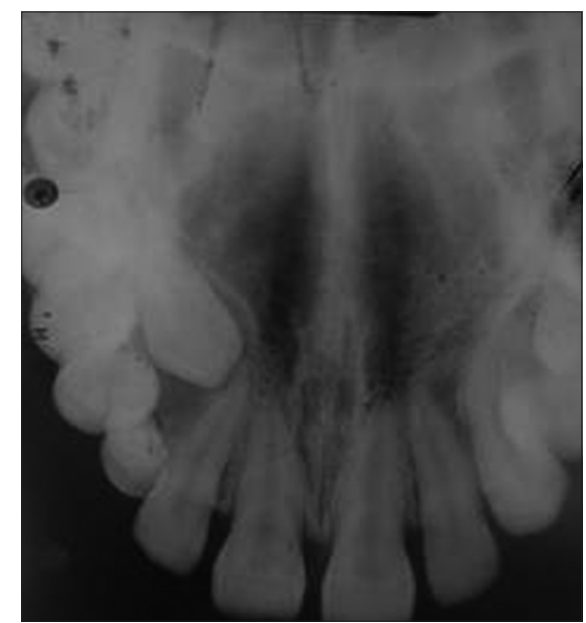

Figure 4: Occlusal radiograph showing retained deciduous canine \& impacted canine

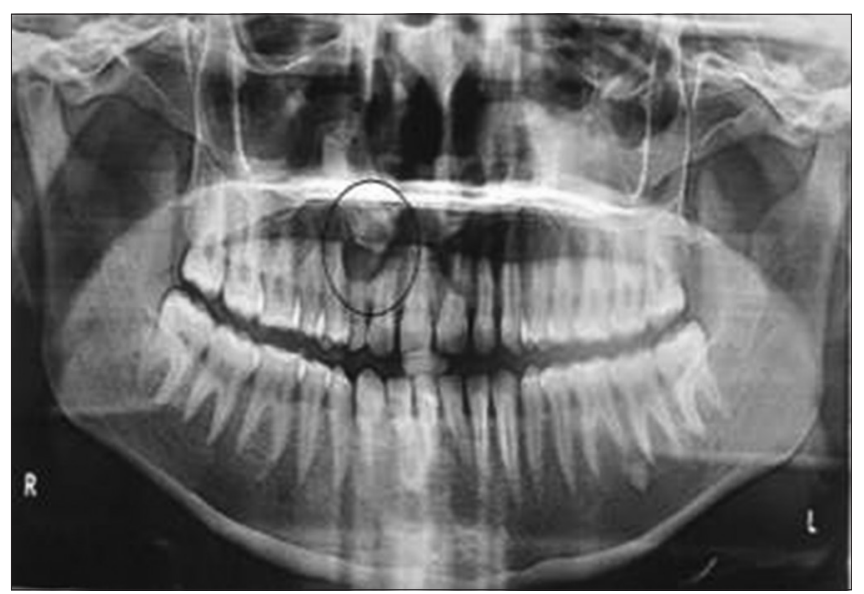

Figure 5: OPG showing periapical radiolucency with deciduous canine \& impacted canine. The extraction of 53 and curettage of extraction socket was done. The soft tissue pieces obtained, were sent for histopathological examination, with a provisional diagnosis of radicular cyst

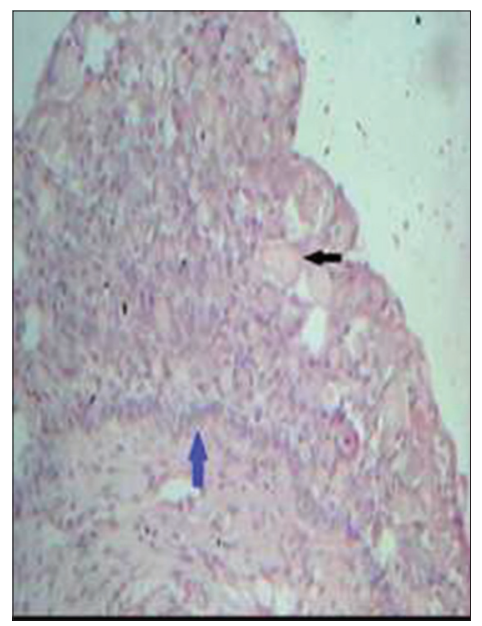

Figure 6: Photomicrograph showing numerous ghost cells lying within the epithelium (H \& E x10) 




Figure 7: High power view showing individual ghost cells (H \& E x40)

was arrived.

\section{DISCUSSION}

Jaws are the most common site for the occurrence of epithelial-lined cysts which are derived from remnants of odontogenic apparatus. Odontogenic cysts can as either be of developmental or inflammatory origin. ${ }^{7}$ The calcifying odontogenic cyst is a rare lesion of the jaws first described as a distinct entity by Gorlin et al., in $1962 .{ }^{5}$ The condition is also referred as Gorlin's cyst, keratinizing ameloblastoma or melanotic ameloblastic odontoma. ${ }^{5}$ The COC represents about $1 \%$ of jaw cysts, ${ }^{6}$ the calcifying odontogenic cyst (COC) is a developmental odontogenic cyst and its occurrence constitute about $0.3-0.8 \%$ of all odontogenic cysts, ${ }^{1} \& 0.37 \%$ to $2.1 \%$ of all odontogenic tumor. $^{7}$

COC, is considered a unique entity with both cystic and neoplastic behaviour. ${ }^{5}$ Cystic variant compromises $85 \%$ of cases. ${ }^{9}$ Clinically CGOC may present either as central $85 \%$ or peripheral lesion $15 \% .{ }^{9}$ Both the intraosseous and extraosseous forms occur, with about equal frequency in the maxilla and mandible, mainly in the incisor and canine areas. Although, the peripheral COC affects individuals in the sixth decade of life, the central variant is observed most commonly in the second decade and does not show gender predilection. ${ }^{6}$ Mostly cases are intraosseous and affects maxilla and mandible with equal frequency, predilection for anterior segment. ${ }^{8}$ Peripheral cases are rarely seen and comprises $13-21 \%$ of all cases. ${ }^{6}$ Our case also shows the location in anterior region of maxilla \& associated with retained deciduous canine. The age of occurrence varies from 3 years to 80 years with definite peaking in the second decade of life. COC can be seen involving a wide age group which lies between 3 years to 80 years with definitive peaking in the $2^{\text {nd }}$ decade. ${ }^{10} \mathrm{COC}$ is often referred as an asymptomatic slow growing swelling of jaws. It is a well circumscribed, solid or cystic lesion derived from odontogenic epithelium or remnants of odontogenic epithelium in the follicle, gingival tissue or bone. ${ }^{5}$

Radiographically, the lesion appears as a unilocular or multilocular well defined radiolucency that may contain small irregular calcified bodies of varying sizes, and it may be associated with an odontoma or an unerupted tooth. ${ }^{6}$ Majority of the lesions present as unilocular form, while in $5-13 \%$ of the cases they are multilocular. They have scattered irregular sized calcification producing a variable range of opacities (salt \& pepper type of patterns). They may be associated with tooth like densities in $50 \%$ of the cases and one third of the cases show association with unerupted tooth, most often canine. ${ }^{4}$ The case reported, also showed a well defined unilocular radiolucency associated with retained deciduous canine and the occlusal radiograph shows impacted canine.

The histological features of a classic calcifying odontogenic cyst are characteristic and present few diagnostic problems. The microscopical feature of a classical COC includes a fibrous capsule with a lining of odontogenic epithelium. The basal layer is made up of ameloblast like columnar or cuboidal cells of 4-10 cell thickness over lined by a loosely arranged epithelial cells having similarity to stellate reticulum of enamel organ. There exists varying number of epithelial cells devoid of nuclei, which are eosinophillic and retain their basic cell outline (ghost cells), these ghost cells may undergo calcification and lose their cellular outline to form sheets like area, of calcified keratin. Ghost cells may be due to effect of coagulative necrosis and dystrophic calcification or it may be a form of normal or abnormal keratinization of the odontogenic epithelium. Ghost cells are not unique to COC, but are also seen in odontoma, ameoblastoma, craniopharyngioma, and other odontogenic tumors and can undergo calcification, which is believed to be dystrophic in nature. The ability to induce dental hard tissue formation appears to be a property of epithelial cell lining of the COC. Hallmark of COC is presence of ghost cells in the cystic epithelium. There are three types of COCs based on histological features, Type 1: Simple monostotic type with the presence of ghost cells with or without dentinoid calcified tissue, Type 2: Formation of calcified tissues in the lumen of the cyst wall, Type 3: Ameloblast like proliferation in the connective tissue and lumen of the cyst may be seen. ${ }^{5}$ 
COC may be an incidental radiological finding \& usually does not present with any symptoms. ${ }^{6}$ Thus keeping all the clinical, radiographical and histopathological features seen, we arrived at a diagnosis of Type 1 COC.

Since the description in 1962 under the term calcifying odontogenic cyst, disagreements exists regarding the nature, terminology and classification, these controversies and confusion about the lesion are due to existence of two variants of the lesion: cystic and neoplastic forms. Some authors consider a "dualistic" concept (lesion existing in two forms either cyst or neoplasm). ${ }^{9}$ Lastly, COC is a lesion which has little tendency to recur and so, enucleation is the treatment of choice. Many cases of recurrence have been reported, Wright et al reported recurrence which has developed 5 years or more after initial treatment. McGowan \& Browne suggested that, a follow-up period of 10 years is advisable. ${ }^{6}$

In 1981, Praetorius et al, framed a classification based on dualistic concept in which they divided COC (as it was called then) into two entities: A cyst and a neoplasm and suggested the term dentogenic ghost cell tumour (DGCT) for the neoplastic variant. Buchner (1991) classified COC into peripheral and central COC, and further sub classified each into cystic or neoplastic variants and included a rare malignant variant of COC in the classification. Hong et al (1991) divided COC into cystic and neoplastic type. Cystic type is again classified into proliferative, nonproliferative, ameloblastomatous and odontoma associated. WHO in 1971 used the term COC describing as non-neoplastic cystic lesion. In 1992 WHO classified this lesion under odontogenic tumor but continued to use the term calcifying odontogenic cyst. As the terminology was misleading and did not explain the complete behaviour of the lesion, in 20005, WHO again renamed the lesion as calcifying cystic odontogenic tumor."

Inadvertent use of the term COC (Gorlin 1962) for the lesions carries the possibility of masking the real biological behaviour of the solid neoplastic variant and neoplastic with cystic architecture, which has high proliferating index, on the other hand use of the term CCOT (WHO 2005) for the lesion may result in unwanted extensive surgical procedure for cystic subtypes. The use of nomenclature should emphasize on biological behaviour of the lesion rather than familiar or older terms, also that the lesion can be approached and treated accordingly.

\section{CONCLUSION}

For better understanding of the incidence, biologic behaviour, recurrence and treatment of a lesion, its presentation with its terminologies, clinical behaviour $\&$ histological description should be encouraged. COC is a unique lesion which posses both cystic and neoplastic variant with different clinical, radiographical and histological characteristics. So, a careful evaluation of its different variants may lead to a definitive diagnosis and treatment planning for the lesion.

\section{REFERENCES}

1. Balaji SM and Rooban T. Calcifying odontogenic cyst with atypical features. Ann Maxillofac Surg, 2012; 2(1) 82-85.

2. Toida M. So-called calcifying odontogenic cyst: review and discussion on the terminology and classification. J Oral Pathol Med 1998; 27:49-52.

3. Takeda Y, Suzuki A and Yamamoto H. Histopathologic study of epithelial components in the connective tissue wall of unilocular type of calcifying odontogenic cyst. J Oral Pathol Med 1990; 19: 108-113.

4. Rajkumar K, Kamal K, Sathish MR and Leena S. Calcifying odontogenic cyst. J Oral Maxillofac Pathol 2004;8(2):99-103.

5. Guruprasad Y, Chouhan D S, Kumar V and Hunsagi P. Calcifying odontogenic cyst of anterior maxilla with complex odontoma. Med J Dr D.Y. Patil Univ 2014; 7(4): 508-512.

6. Aswath N, Mastan K, Manikandan T and Samuel G. Odonto calcifying cyst. Contemp Clin Dent 2013; 4(1): 108-111.

7. Nayak S, Shukla $P$, Jose $M$ and Rao Sripathi $B H$. Ameloblastomatous calcifying odontogenic cyst in the mandible - A case report of a rare histological variant. J Adv Med Dent Scie 2014; 2(2):123-129.

8. Kler S, Palaskar S, Shetty VP and Bhushan A. Intraosseous calcifying cystic odontogenic tumor. JOMFP 2009; 13(1): 27-29.

9. Thinakaran $M$, Sivakumar $P$, Ramalingam $S$, Jeddy $N$ and Balaguhan S. Calcifying ghost cell odontogenic cyst: A review on terminologies and classifications. JOMFP 2012; 16(3): 450-453.

10. Narayanan A, Acharya SS, Patnaik $S$ and Akheel M. Calcifying epithelial odontogenic cyst in maxilla: A case report. IJSS Case Reports \& Reviews 2014; 1(2):10-12.

\section{Authors Contribution:}

BNS - Reviewed the manuscript, Editing of the manuscript, confirmation of diagnosis of the case; SR - Review of the case report, constructive criticism and editing of the manuscript; BN - diagnosis of the case report, format of presentation; NR - preparation of manuscript, review and final approval.

Source of Support: Nil, Conflict of Interest: None declared. 